\title{
A CHARACTERIZATION OF CERTAIN CONFORMALLY EUCLIDEAN SPACES OF CLASS ONE
}

\author{
G. M. LANCASTER
}

1. In this paper we will examine the metrics of conformally Euclidean spaces $C_{n}(n \geqq 4)$ having the following two properties:

(1) They are locally and isometrically imbeddable in Euclidean space of one higher dimension $\left(E_{n+1}\right)$, i.e. they are of class one.

(2) With respect to a conformal coordinate system, the matrix of the second fundamental tensor $\left[b_{i j}\right]$ has diagonal form.

The condition for class one is that there exist a (second fundamental) tensor $\left[b_{i j}\right]$, satisfying the Gauss (1.1) and Codazzi (1.2) equations:

$$
\begin{aligned}
R_{h i j k} & =b_{h j} b_{i k}-b_{h k} b_{i j}, \\
b_{i j, k} & =b_{i k, j} .
\end{aligned}
$$

To satisfy (2), we will therefore look for a solution of these equations for which $b_{i j}=0$ when $i \neq j$.

Sen, in a series of papers ([4], [5] and [6]), has investigated certain conditions for a $C_{n}$ to be of class one, and obtained [6, Theorem 3] a canonical form for the metric of such a space. His result, however, is incorrect in its full generality (see [3] for a disproof). In 1962, at the meeting of the International Congress of Mathematicians in Stockholm [8], R. Blum presented, without proof, a canonical form for the metric of a $C_{n}$ satisfying (1) and (2) above and such that $n \geqq 4$. In his theorem, however, Blum overlooked an exception, and it is therefore not correct as stated. It is the purpose of this paper to give a proof and a simplification of the corrected result.

Thomas [7] showed that when $\tau$, the rank of the matrix $\left[b_{i j}\right]$, is greater than or equal to four, equations (1.2) follow as a consequence of equations (1.1). It is therefore logical to consider separately the cases $n \geqq 4$ and $n=3$ (the case $n=2$ is not considered here; the surfaces $\bar{C}_{2}^{1}$ are called isothermal surfaces and form a separate area of study in themselves). It will turn out that for $n \geqq 4, \tau$ is greater than or equal to four except in two particular cases. In both, however, it is easily verified that equations (1.2) are satisfied because of (1.1). For $n=3$, the situation is somewhat different and the Codazzi equations must be considered separately as a set of independent conditions. This case will be the object of investigation in a later paper.

Received by the editors August 17, 1967. 
As regards notation, a $C_{n}$ having property (1) will be denoted $C_{n}^{\mathbf{1}}$, following Sen's example, while a $C_{n}$ which has both properties (1) and (2) will be denoted $\bar{C}_{n}^{1}$. Tensor notation used throughout will be essentially that to be found in Eisenhart [2].

2. Referred to a conformal coordinate system, the metric of a $C_{n}$ is

$$
d s^{2}=e^{2 \sigma} \sum_{i}\left(d x^{i}\right)^{2}
$$

where $\sigma=\sigma\left(x^{1}, x^{2}, \cdots, x^{n}\right)$ and $e^{-2 \sigma} \neq 0$.

By routine calculation we then obtain the following expression for the Riemann Curvature Tensor:

$$
R_{h i j k}=e^{2 \sigma}\left[\delta_{h k} \sigma_{i j}+\delta_{i j} \sigma_{h k}-\delta_{h j} \sigma_{i k}-\delta_{i k} \sigma_{h j}+\sum_{m} \sigma_{, m}^{2}\left(\delta_{h j} \delta_{i k}-\delta_{h k} \delta_{i j}\right)\right]
$$

where $\sigma_{i j}=\sigma_{, i j}+\sigma_{, i} \sigma_{, j}$, and $\sigma_{, i j}$ (the second covariant derivative of $\sigma$ ), is given by

$$
\sigma_{, i j}=\partial_{i} \partial_{j} \sigma-2 \sigma_{, i} \sigma_{, j}+\delta_{i j} \sum_{m} \sigma_{, m}^{2}
$$

Substituting this expression into (1.1) and considering components yields the following two equations:

$$
\sigma_{i j}=0 \quad(i \neq j ; j=1, \cdots, n),
$$

and

$$
b_{h h} b_{i i}=e^{2 \sigma}\left(\sum_{m} \sigma_{, m}^{2}-\sigma_{h h}-\sigma_{i i}\right) \quad(h \neq i ; h, i=1, \cdots, n) .
$$

We will now consider each of these relations in turn.

3. Equation (2.2) simplifies to

$$
\partial_{i} \partial_{j} \sigma-\partial_{i} \sigma \partial_{j} \sigma=0 \quad(i \neq j) .
$$

If we now multiply this by $e^{-\sigma}$ we obtain

$$
\partial_{i} \partial_{j} e^{-\sigma}=0 \quad(i \neq j) .
$$

Thus

$$
\partial_{j} e^{-\sigma}=F\left(x^{j}\right) \quad(j=1,2, \cdots, n),
$$

and hence

$$
e^{-\sigma}=\sum_{m} f_{m}
$$

where $f_{m}$ is a function of $x^{m}$ only. 
4. Utilizing equation (3.1), (2.3) reduces to

$$
b_{h h} b_{k k}=e^{4 \sigma}\left[e^{-\sigma}\left(f_{h}^{\prime \prime}+f_{k}^{\prime \prime}\right)-A\right] \quad(h \neq k),
$$

where

$$
A=\sum_{m} f_{m}^{\prime 2}
$$

Similarly

$$
b_{i i} b_{j j}=e^{4 \sigma}\left[e^{-\sigma}\left(f_{i}^{\prime \prime}+f_{j}^{\prime \prime}\right)-A\right] \quad(i \neq j) .
$$

Multiplying (4.1) and (4.3) we obtain

$$
\begin{aligned}
b_{h h} b_{k k} b_{i i} b_{j j}=e^{8 \sigma}\left[e ^ { - 2 \sigma } \left(f_{h}^{\prime \prime}\right.\right. & \left.+f_{k}^{\prime \prime}\right)\left(f_{i}^{\prime \prime}+f_{j}^{\prime \prime}\right) \\
& \left.-A e^{-\sigma}\left(f_{h}^{\prime \prime}+f_{k}^{\prime \prime}+f_{i}^{\prime \prime}+f_{j}^{\prime \prime}\right)+A^{2}\right] \\
& (h \neq k, i \neq j),
\end{aligned}
$$

and similarly:

$$
\begin{aligned}
b_{h h} b_{i i} b_{k k} b_{j j}=e^{8 \sigma}\left[e ^ { - 2 \sigma } \left(f_{h}^{\prime \prime}\right.\right. & \left.+f_{i}^{\prime \prime}\right)\left(f_{k}^{\prime \prime}+f_{j}^{\prime \prime}\right) \\
& \left.-A e^{-\sigma}\left(f_{h}^{\prime \prime}+f_{i}^{\prime \prime}+f_{k}^{\prime \prime}+f_{j}^{\prime \prime}\right)+A^{2}\right] \\
& (h \neq i, k \neq j) .
\end{aligned}
$$

Equate (4.4) and (4.5) and simplify. Then

$$
\left(f_{i}^{\prime \prime}-f_{k}^{\prime \prime}\right)\left(f_{j}^{\prime \prime}-f_{h}^{\prime \prime}\right)=0 \quad(i \neq h, i \neq j, k \neq j, k \neq h) .
$$

From this expression, we then deduce the result that $f_{i}^{\prime \prime}=2 a$ (constant) for all $i$ except one value, say $i=1$. Thus

$$
f_{i}=a x^{i^{2}}+b_{i} x^{i}+c_{i} \quad(i=2,3, \cdots, n)
$$

while $f_{1}$ is arbitrary.

Putting $f=f_{1}+\sum_{i=2}^{n} c_{i}$ and substituting (4.6) and (3.1) into (2.1) we thus obtain

$$
d s^{2}=\frac{\sum_{i=1}^{n}\left(d x^{i}\right)^{2}}{\left[f\left(x^{1}\right)+a \sum_{m=2}^{n}\left(x^{m}\right)^{2}+\sum_{m=2}^{n} b_{m} x^{m}\right]^{2}} .
$$

It is then a fairly straightforward matter to obtain explicit expressions for the $b_{i i}$ from equation (4.3), viz

$$
b_{i i}=e^{2 \sigma}\left(4 a f-f^{\prime 2}-\sum_{m=2}^{n} b_{m}^{2}\right)^{1 / 2} \quad(i=2,3, \cdots, n),
$$


and

$$
\begin{aligned}
b_{11} & =b_{i i}+\frac{e^{3 \sigma}\left(f^{\prime \prime}-2 a\right)}{b_{i i}} \quad \text { if } b_{i i} \neq 0 \quad(i \neq 1), \\
& =0 \quad \text { if } b_{i i}=0 \quad(i \neq 1) .
\end{aligned}
$$

However, there is an interesting exception which arises when $b_{i i}=0(i=2, \cdots, n)$ and $a \neq 0$. If we equate equation (4.8) to zero and solve, we obtain the following two independent solutions:

$$
\begin{aligned}
& f\left(x^{1}\right)=a x^{1^{2}}+b_{1} x^{1}+\sum_{m=1}^{n} b_{m}^{2} / 4 a \\
& f\left(x^{1}\right)=\sum_{m=2}^{n} b_{m}^{2} / 4 a .
\end{aligned}
$$

Solution (1) implies that $\bar{C}_{n}^{1}$ is a Euclidean space and hence $b_{11}=0$ also, as indicated in equation (4.9).

Solution (2), however, yields a contradiction to the effective Gauss equations (4.1), and hence represents a space which is not a $\bar{C}_{n}^{1}$. We may see this by direct substitution into equations (4.1);

$$
\begin{array}{rll}
h, i \neq 1 & \text { yields } & 4 a e^{-\sigma}-A=0, \\
h=1, i \neq 1 & \text { yields } & 2 a e^{-\sigma}-A=0,
\end{array}
$$

and together these imply $e^{-\sigma}=0$, i.e. a contradiction.

Furthermore, the space $C_{n}$, whose metric is given by equation (4.7) with $f\left(x^{1}\right)=\sum_{m=2}^{n} b_{m}^{2} / 4 a$, is not even of class one, i.e. a $C_{n}^{1}$. This may be seen by obtaining the components of the curvature tensor from this metric and looking for a solution $\left[b_{i j}\right]$, not necessarily diagonal, to equations (1.1). A contradiction is thereby obtained. The space is in fact of class two (see [1]).

Thus to equation (4.7) we must add the condition that $f\left(x^{1}\right)$ $\neq \sum_{m=2}^{n} b_{m}^{2} / 4 a$.

5. The Codazzi equations follow because of Thomas' result, except for the following situations:

(a) $b_{11}=b_{i i}=0(i=2, \cdots, n)$, in which case they are satisfied identically.

(b) $b_{11}=0, b_{i i} \neq 0(i=2, \cdots, n)$, and $n=4$. This situation occurs when $b_{i i}^{2}=e^{3 \sigma}\left(2 a-f^{\prime \prime}\right)(i=2,3,4)\left(f^{\prime \prime} \neq 2 a\right)$, and $f$ satisfies the differential equation

$$
f\left(f^{\prime \prime}+2 a\right)-f^{\prime 2}+\left(f^{\prime \prime}-2 a\right) \sum_{m=2}^{4}\left(a x^{m^{2}}+b_{m} x^{m}\right)-\sum_{m=2}^{4} b_{m}^{2}=0 .
$$


It can be fairly readily verified that here again the Codazzi equations are satisfied (by converting these equations to the simpler form

$$
\partial_{k} b_{i i}=\sigma_{, k}\left(b_{i i}+b_{k k}\right) \quad(i \neq k)
$$

and checking all the cases).

6. Conversely, if we are given a $C_{n}$ with metric (4.7), and $f\left(x^{1}\right)$ $\neq \sum_{m=2}^{n} b_{m}^{2} / 4 a$, we may construct a tensor $\left[b_{i j}\right]$ using equations (4.8) and (4.9) such that $b_{i j}=0$ for $i \neq j$. These in turn satisfy the Gauss and Codazzi equations. Furthermore, the tensor $\left[b_{i j}\right]$ is unique except for sign provided that rank $\left[b_{i j}\right](=\tau) \geqq 3$ (see $[7$, p. 188]). This is always true unless $C_{n}$ is a Euclidean space (in which case it is of class zero anyway).

7. We can further simplify the metric (4.7) by considering separately the cases when $a=0$ and $a \neq 0$.

$a \neq 0$. The transformation $y^{1}=a x^{1}, y^{m}=a x^{m}+b_{m} / 2(m=2,3, \cdots$, $n$ ) changes the metric to the simpler form

(7.1) $d s^{2}=\frac{\sum_{i=1}^{n}\left(d y^{i}\right)^{2}}{\left[F\left(y^{1}\right)+\theta\right]^{2}} \quad$ where $\theta=\sum_{i=2}^{n}\left(y^{i}\right)^{2}$ and $F\left(y^{1}\right) \neq 0$.

$a=0$. Here if $b_{m}(m=2,3, \cdots, n)$ are all zero, we obtain the metric

$$
d s^{2}=\frac{\sum_{i=1}^{n}\left(d x^{i}\right)^{2}}{\left[f\left(x^{1}\right)\right]^{2}}
$$

whereas if the $b_{m}$ are not all zero, we may make any orthogonal transformation such that

$$
y^{1}=x^{1}, \quad y^{2}=\sum_{m=2}^{n} \frac{b_{m}}{B} x^{m}, \quad \text { where } \quad B=\left(b_{2}^{2}+b_{3}^{2}+\cdots+b_{n}^{2}\right)^{1 / 2},
$$

and obtain the metric

$$
d s^{2}=\frac{\sum_{i=1}^{n}\left(d y^{i}\right)^{2}}{\left[f\left(y^{1}\right)+B y^{2}\right]^{2}}
$$

Thus in both cases when $a=0$, the metric of a $\bar{C}_{n}^{1}$ reduces to the form 


$$
d s^{2}=\frac{\sum_{i=1}^{n}\left(d x^{i}\right)^{2}}{\left[f\left(x^{1}\right)+K x^{2}\right]^{2}}
$$

where $K$ is an arbitrary constant.

8. The following theorem summarizes the results obtained in the preceding sections:

THEOREM. Let $\bar{C}_{n}^{1}(n \geqq 4)$ be a conformally Euclidean space of class one, such that, with respect to a conformal coordinate system $x^{1}, x^{2}$, $\cdots, x^{n}$, the second fundamental tensor has diagonal form. Then the metric of $\bar{C}_{n}^{1}$ takes one of the following two distinct canonical forms:

$$
\begin{aligned}
d s^{2} & =\frac{\sum_{i=1}^{n}\left(d x^{i}\right)^{2}}{\left[f\left(x^{1}\right)+\theta\right]^{2}} \text { where } \theta=\sum_{i=2}^{n}\left(x^{i}\right)^{2}, \\
d s^{2} & =\frac{\sum_{i=1}^{n}\left(d x^{i}\right)^{2}}{\left[g\left(x^{1}\right)+K x^{2}\right]^{2}},
\end{aligned}
$$

where $f$ and $g$ are arbitrary twice differentiable functions of $x^{1}$ only, except that $f\left(x^{1}\right) \neq 0$, and $K$ is an arbitrary constant.

Conversely, if a $C_{n}(n \geqq 4)$ possesses either of the metrics (I) or (II), then it is a $\bar{C}_{n}^{1}$.

\section{REFERENCES}

1. H. W. Brinkman, On Riemannian spaces conformal to Euclidean spaces, Proc. Nat. Acad. Sci. U.S.A. 9 (1923), 1-3.

2. L. P. Eisenhart, Riemannian geometry, 5th printing, Princeton Univ. Press, Princeton, N. J., 1964.

3. G. M. Lancaster, Note on a theorem by Sen, Proc. Amer. Math. Soc. 19 (1968), 1119-1122.

4. R. N. Sen, On a type of Riemannian space conformal to a flat space, J. Indian Math. Soc. 21 (1957), 105-114.

5. - Conformally Euclidean space of class one, Indian J. Math. 6 (1964), 93-103.

6. - On conformally-flat Riemannian space of class one, Proc. Amer. Math. Soc. 17 (1966), 880-883.

7. T. Y. Thomas, Riemannian spaces of class one and their characterization, Acta Math. 67 (1936), 169-211.

8. International Congress of Mathematicians, Abstract of short communications, Stockholm 1962, Almquist and Wiksells, Uppsala, 1962.

UNIVERSITY OF SASKATCHEWAN 